\title{
AN IMPROVED BOND-BASED PERIDYNAMIC MODEL BASED ON TIMOSHENKO BEAM THEORY
}

\author{
XI-ZHUO CHEN', FENG-JUN CHEN², XIN-XIN WANG ${ }^{2}$, WEN SHEN² AND HAI- \\ TAO YU ${ }^{3}$
}

${ }^{1}$ Department of Geotechnical Engineering Tongji University, Shanghai 200092, China

2Shanghai Construction Group Co., Ltd Shanghai 200080, China

${ }^{3}$ Key Laboratory of Geotechnical and Underground Engineering of Ministry of Education Tongji University, Shanghai 200092, China yuhaitao@tongji.edu.cn

Key words: Peridynamic model, Bond-based, Timoshenko beam, Failure criterion.

\begin{abstract}
An improved constitutive model based on Timoshenko beam theory is proposed for bond-based peridynamics. The motion and force governing equations of the bond are established by introducing Timoshenko beam element to simulate the interaction between the particles including the bond tension-rotation-shear coupling effects. Since the axial displacement, transverse displacement and relative rotation angle of the bond are considered in the model, it can overcome the limitation of Poisson's ratio in the classical bond-based peridynamics model. Three kinds of peridynamic parameters, corresponding to the compressive, shear and bending stiffness of the bond, are introduced to keep the consistence between the strain energy of the peridynamic model and that of the continuum mechanics under arbitrary deformation field. Moreover, an energy-based failure criterion, involving the maximum stretch, shear strain and rotation angle limits of the bond, is proposed to capture the progressive failure of general quasi-brittle materials. The validation of the proposed model is verified by comparing the simulation results to the experiment observations and analytical solution. Numerical results show that this improved model can be widely used to predict the nonlinear deformation, crack propagation and progressive failure of materials with variable Poisson's ratio under complex loading conditions.
\end{abstract}

\section{INTRODUCTION}

In order to overcome the basic incompatibility of cracks with the partial differential equations, originated from the classical theory of solid mechanics, Silling [1] proposed the peridynamic theory, which formulates mechanical problems based on integral equations instead of partial differential equations. Different kinds of peridynamic models, including bond-based and state-based peridynamic models [2,3], are proposed in current study. Compared with the state-based peridynamic model, the bond-based peridynamic (BB-PD) is 
simpler in modelling and more stable in crack development simulation. However, the classical BB-PD model has the limitation for the Poisson's ratio.

Some researchers have made significant efforts to address this problem. Gerstle et al. [4] developed a micropolar BB-PD model by introducing the pairwise moment into the interacting force between the particles, which can simulate materials with different Poisson's ratio. Then, a linear elastic BB-PD model is proposed by Prakash and Seidel [5] to solve the restriction of the Poisson's ratio. Based on the modified Stillinger-Weber potential functions, the conjugated BB-PD model [6] is proposed to improve the simulation accuracy for fracture problems. Zhu and Ni [7] developed an enriched BB-PD model to couple the bond rotation effect, which is suitable for the simulation of elastic isotropic materials with varying Poisson's ratios. Diana and Casolo [8] extended the micropolar BB-PD model to capture the particle relative rotation angle, and it was validated for simulating the behaviors of solids with variable Poisson's ratios under the non-homogeneous deformation field.

Generally, the consistence criterion of the energy between the peridynamics and the continuum mechanics is the key factor to guarantee the simulation accuracy of the model $[9,10]$. However, the energy for current BB-PD models is only consistent with that of continuum mechanics under simple loads, such as pure tension and pure shearing, which will cause the imbalance of the energy for complex dynamic analysis. This is due to the fact that, in these BB-PD models, the mechanical behaviors of particles are normally simulated by independent springs ignoring the tension-rotation-shearing coupling effect. On the other hand, the failure criteria of current BB-PD models are only used for the simulation of linear elastic brittle materials, but failed to simulate the progressive failure process of the quasi-britte materials. The purpose

This paper provide

rotation and shearing interaction between the compressive, shear and bending stiffnesses of the beam, are introduced to describe the

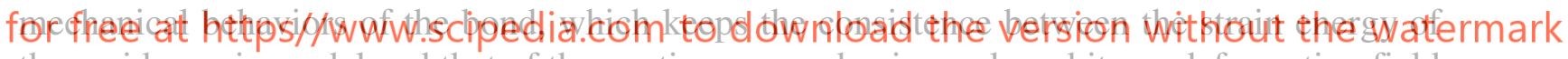
the peridynamic model and that of the continuum mechanics under arbitrary deformation field. A new energy-based failure criterion is proposed to describe progressive failure process of quasi-brittle materials. The comparisons between the simulation results with those of experimental observations and analytical solutions are performed to verify the validation of the improved BB-PD model.

\section{THE IMPROVED BOND-BASED PERIDYNAMIC MODEL}

Different from the Euler-Bernoulli beam used in micropolar BB-PD models [4], the Timoshenko beam is introduced in the proposed model to simulate the interaction between the particles, which can consider both the shear deformation and rotational inertia of the bond.

\subsection{Motion and force governing equations}

Based on the Timoshenko beam theory, the motion governing equations of the proposed model can be obtained as 


$$
\begin{gathered}
\rho \ddot{\mathbf{u}}\left(\mathbf{x}_{i}, t\right)=\int_{H_{x}} \mathbf{f}\left(\boldsymbol{\eta}, \boldsymbol{\xi}, \boldsymbol{\theta}_{i}, \boldsymbol{\theta}_{j}\right) d V_{j}+b\left(\mathbf{x}_{i}, t\right) \\
(\rho I / A) \ddot{\boldsymbol{\theta}}\left(\mathbf{x}_{i}, t\right)=\int_{H_{x}} \mathbf{m}\left(\boldsymbol{\eta}, \boldsymbol{\xi}, \boldsymbol{\theta}_{i}, \boldsymbol{\theta}_{j}\right) d V_{j}+n\left(\mathbf{x}_{i}, t\right)
\end{gathered}
$$

where $\boldsymbol{\theta}_{i}$ and $\boldsymbol{\theta}_{j}$ are the relative rotation angles of the particles $\mathbf{x}_{i}$ and $\mathbf{x}_{j} ; \ddot{\theta}\left(\mathbf{x}_{i}, t\right)$ is the angular acceleration of the particle $\mathbf{x}_{i} ; \mathbf{m}\left(\boldsymbol{\eta}, \boldsymbol{\xi}, \boldsymbol{\theta}_{i}, \boldsymbol{\theta}_{j}\right)$ and $n\left(\mathbf{x}_{i}, t\right)$ are the internal moment and external moment exerted on the particle $\mathbf{x}_{i}$, respectively.

Introducing three peridynamic parameters, related to the stretch, shear and bending stiffness of the Timoshenko beam, one can obtain the force governing equations of the bond as

$$
\hat{f}_{x}=\left(C_{N} / \xi\right) \hat{u}_{x}, \hat{f}_{y}=\left(12 C_{\theta} / \xi^{3}\right) \hat{u}_{y}+\left(6 C_{\theta} / \xi^{2}\right) \hat{\theta}_{z}, \hat{f}_{z}=\left(12 C_{\theta} / \xi^{3}\right) \hat{u}_{z}-\left(6 C_{\theta} / \xi^{2}\right) \hat{\theta}_{y}
$$

$$
\hat{m}_{y}=\left(6 C_{\theta} / \xi^{2}\right) \hat{u}_{z}-\left(4 C_{M} / \xi\right) \hat{\theta}_{y}, \hat{m}_{z}=-\left(6 C_{\theta} / \xi^{2}\right) \hat{u}_{y}-\left(4 C_{M} / \xi\right) \hat{\theta}_{z}
$$

\subsection{Strain energy and peridynamic parameters}

\section{Integrating the potential function $w$ over the horizon $H_{x}$, the strain energy density $W_{P D}$ of} the proposed model can be derived as
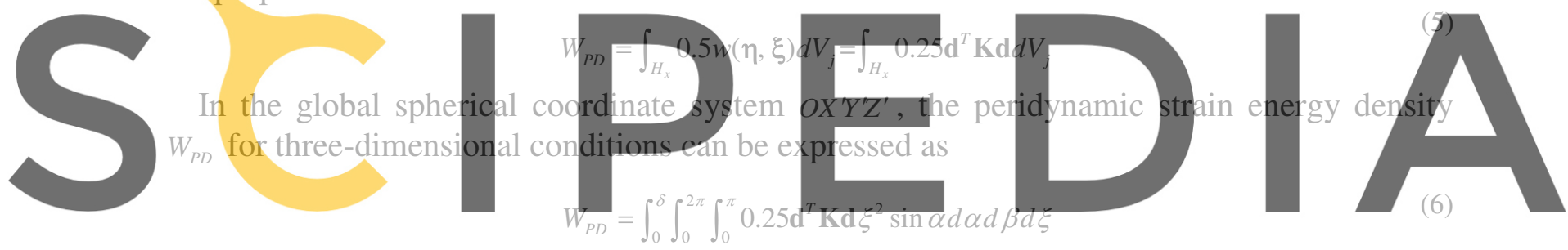

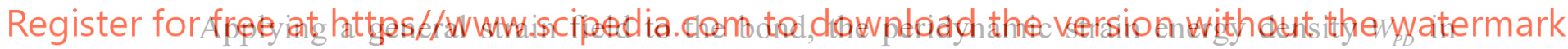

global coordinate system can be obtained as

$$
W_{P D}=\frac{1}{4}\left\{\begin{array}{c}
\varepsilon_{x}^{\prime} \\
\varepsilon_{y}^{\prime} \\
\varepsilon_{z}^{\prime} \\
\gamma_{y}^{\prime} \\
\gamma_{z}^{\prime}
\end{array}\right\}^{T}\left[\begin{array}{ccccc}
k_{11} & k_{12} & k_{13} & 0 & 0 \\
k_{21} & k_{22} & k_{23} & 0 & 0 \\
k_{31} & k_{32} & k_{33} & 0 & 0 \\
0 & 0 & 0 & k_{44} & 0 \\
0 & 0 & 0 & 0 & k_{55}
\end{array}\right]\left\{\begin{array}{c}
\varepsilon_{x}^{\prime} \\
\varepsilon_{y}^{\prime} \\
\varepsilon_{z}^{\prime} \\
\gamma_{y}^{\prime} \\
\gamma_{z}^{\prime}
\end{array}\right\}
$$

where

$$
\begin{aligned}
& k_{11}=k_{22}=k_{33}=(1 / 5) C_{N} \delta^{4} \pi+(16 / 5) C_{\theta} \delta^{2} \pi, \quad k_{44}=k_{55}=(1 / 15) C_{N} \delta^{4} \pi-(8 / 5) C_{\theta} \delta^{2} \pi+8 \pi C_{M} \delta^{2}, \\
& k_{12}=k_{13}=k_{21}=k_{31}=k_{32}=k_{23}=(1 / 15) C_{N} \delta^{4} \pi-(8 / 5) C_{\theta} \delta^{2} \pi
\end{aligned}
$$

Equating the peridynamic strain energy density to the strain energy density in the continuum mechanics, one can obtain the peridynamic parameters for three-dimensional conditions as 


$$
C_{N}=\frac{6 E}{\delta^{4}(1-2 v) \pi} C_{\theta}=\frac{(1-4 v) E}{4 \delta^{2} \pi(1+v)(1-2 v)} C_{M}=\frac{3(1-4 v) E}{16 \delta^{2}(1-2 v)(1+v) \pi}
$$

Similarly, for the plane stress condition, the peridynamic parameters can be derived as

$$
C_{N}=\frac{6 E}{\pi h(1-v) \delta^{3}} C_{\theta}=\frac{E(1-3 v)}{6 \pi h(1-v)(1+v) \delta} C_{M}=\frac{E(1-3 v)}{8 \pi \delta(1-v)(1+v) h}
$$

For the plane stress condition, we can obtain the peridynamic parameters as

$$
C_{N}=\frac{6 E}{\pi h \delta^{3}(1-2 v)(1+v)} C_{\theta}=\frac{E(1-4 v)}{6 \pi h(1-2 v)(1+v) \delta} C_{M}=\frac{(1-4 v) E}{8 \pi \delta(1-2 v)(1+v) h}
$$

\subsection{Failure criteria and local damage variables}

The failure of typical quasi-brittle materials contains linear elastic deformation and progressive damage stages [11]. In order to further capture the progressive failure process of the quasi-brittle materials, a novel energy-based failure criterion, related to the bond stretch, bond shear deformation and rotation angle between particles, is developed in this section.

Since the crack is initiated and propagated in the peridynamic model by breaking every bond across the fracture surface, the fracture surface energy $G_{0}$ should be equal to the strain energy in all the broken bonds, which is expressed in the form as
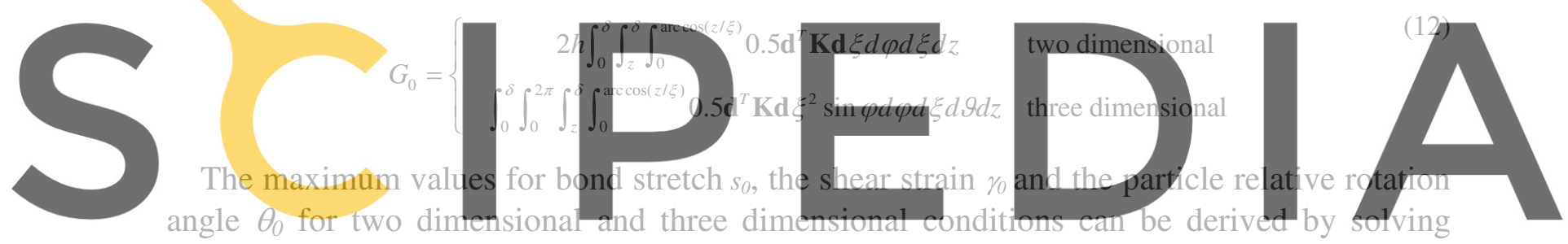

these integrations as

Register for free at https//www.scipedia.com to download the version without the watermark

$$
\begin{aligned}
& s_{0}=\sqrt{\frac{4 G_{0}}{\delta^{4} h C_{N}}} \gamma_{0}=\sqrt{\frac{G_{0}}{6 \delta^{2} h C_{\theta}}} \theta_{0}=\sqrt{\frac{G_{0}}{2 \delta^{2} h C_{M}}} \text { two dimensional } \\
& s_{0}=\sqrt{\frac{10 G_{0}}{\pi C_{N} \delta^{5}}} \gamma_{0}=\sqrt{\frac{G_{0}}{2 \pi C_{\theta} \delta^{3}}} \theta_{0}=\sqrt{\frac{3 G_{0}}{2 \pi C_{\theta} \delta^{3}}} \text { three dimensional }
\end{aligned}
$$

The progressive failure process of the bond between particles can be described by the three scalar valued functions $\mu_{s}(\xi, t), \mu_{\gamma}(\xi, t)$ and $\mu_{\theta}\left(\boldsymbol{\theta}_{j}-\boldsymbol{\theta}_{i}, t\right)$ corresponding to the bond stretch, shear deformation and particle relative rotation angle, respectively.

$$
\mu_{s}(\xi, t)=\left\{\begin{array}{cl}
1 & \text { if }-s_{0}<s<s_{0} \text { for all } t>0 \\
\frac{s_{0}}{s} e^{-k \frac{s-s_{0}}{s_{0}}} & \text { if } s \geq s_{0} \text { for all } t>0 \\
\frac{-s_{0}}{s} e^{k \frac{s+s_{0}}{s_{0}}} & \text { if } s \leq-s_{0} \text { for all } t>0
\end{array}\right.
$$




$$
\begin{gathered}
\mu_{\gamma}(\boldsymbol{\xi}, t)=\left\{\begin{array}{c}
1 \quad \text { if }-\gamma_{0}<\gamma<\gamma_{0} \text { for all } t>0 \\
\frac{\gamma_{0}}{\gamma} e^{-k \frac{\gamma-\gamma_{0}}{\gamma_{0}}} \quad \text { if } \gamma \geq \gamma_{0} \text { for all } t>0 \\
\frac{-\gamma_{0}}{\gamma} e^{k \frac{\gamma+\gamma_{0}}{\gamma_{0}}} \quad \text { if } \gamma \leq-\gamma_{0} \text { for all } t>0
\end{array}\right. \\
\mu_{\theta}\left(\boldsymbol{\theta}_{j}-\boldsymbol{\theta}_{i}, t\right)= \begin{cases}\frac{\theta_{0}}{\theta} e^{-k \frac{\theta-\theta_{0}}{\theta_{0}}} \quad \text { if } \theta \geq \theta_{0} \text { for all } t>0 \\
\frac{-\theta_{0}}{\theta} e^{k \frac{\theta+\theta_{0}}{\theta_{0}}} & \text { if } \theta \leq-\theta_{0} \text { for all } t>0\end{cases}
\end{gathered}
$$

where $k$ is the defined parameter obtained from polyaxial test data in materials, which governs the reduction of forces and moment of the bond. Considering the progressive failure process of the quasi-brittle materials, the limit values for $s_{0}, \gamma_{0}$ and $\theta_{0}$ can be derived by
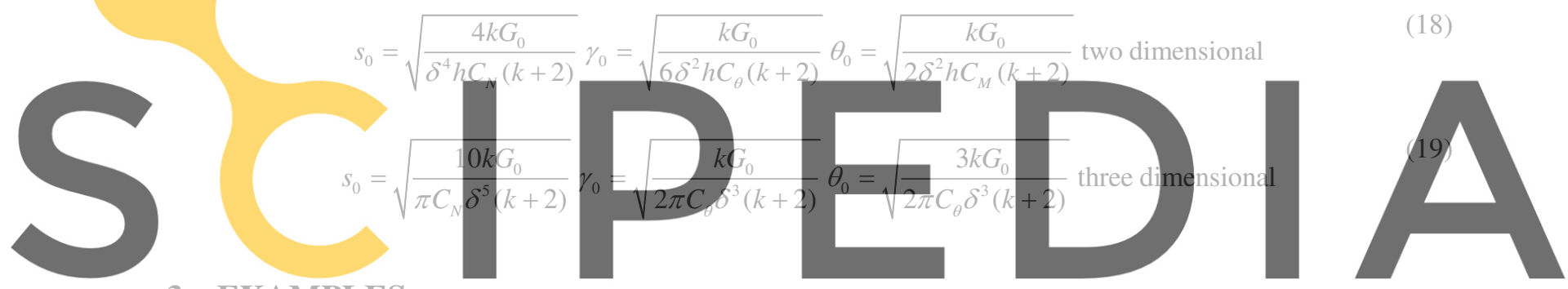

3 EXAMPLES

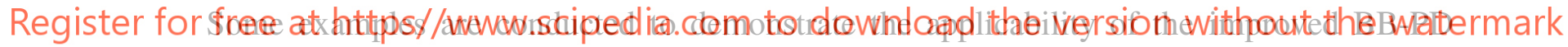
model under tensile and tension-shear combined loads. The validation of the improved BBPD model is verified by comparing its predictions with those of experimental observations and analytical solutions.

\subsection{Tensile loading}

The two dimensional plates with different Poisson's ratios under uniaxial tension, are utilized to verify the elastic behavior of the improved peridynamic model. The rectangular plates with $1 \mathrm{~m}$ length, $0.5 \mathrm{~m}$ width and $0.01 \mathrm{~m}$ thickness are considered. The plates are considered linear isotropic, homogeneous, and elastic, with Young's modulus $E=1.92 \times 10^{11} \mathrm{~Pa}$ and mass density $\rho=8000 \mathrm{~kg} / \mathrm{m}^{3}$. The uniaxial tension loading $P$ imposed on the right and left sides of the plates is $200 \mathrm{MPa}$ along the $\mathrm{x}$-axis. The plates are discretized with a spacing of $\Delta=0.01 \mathrm{~m}$ and the material horizon is selected as $\delta=3 \Delta$. Five different kinds of Poisson's ratio $v$, namely $0.1,0.15,0.2,0.25$ and 0.3 , are selected. Fig. 1 shows the displacements of the plates in y coordinate obtained from the improved BB-PD model and analytical solution. As shown in the figure, the displacements of the plates with varying Poisson's ratio are in quite good agreements with the analytical values, implying that the proposed model enables 
describing elastic behavior of materials with variable Poisson's ratio.

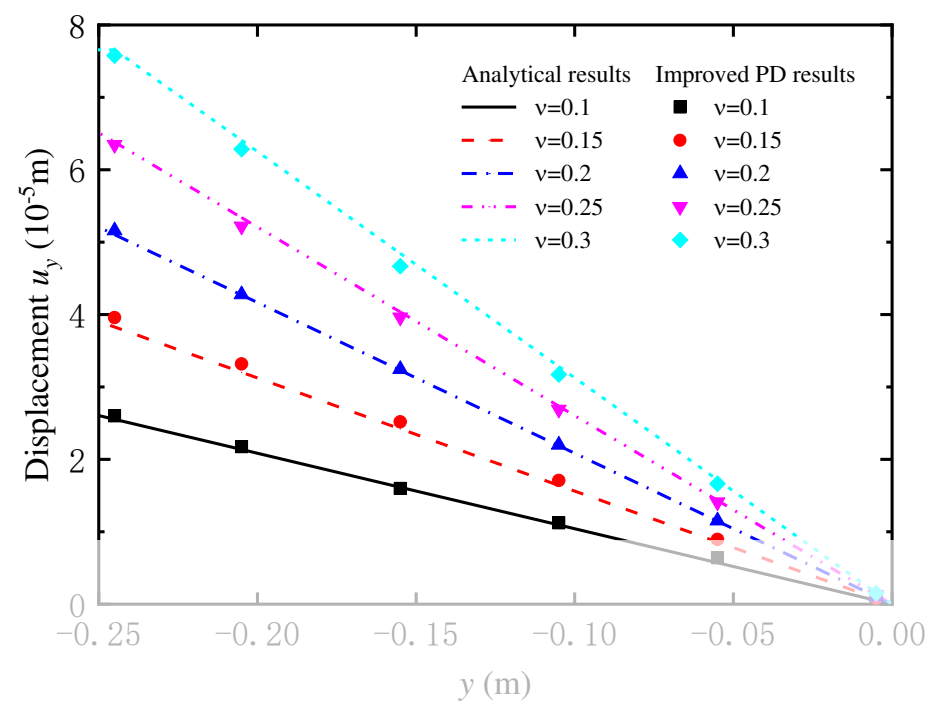

Figure 1: Displacements of the plates with varying Poisson's ratio in the y coordinate.

\subsection{Tension-shear combined loading}

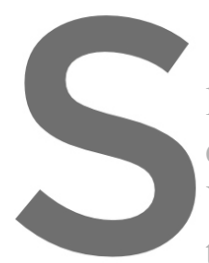

The double-edge-notched loads is used to demonstrate complicated loading Young's modulus E=32Gpa tensile strength $\sigma_{\mathrm{t} 0}=3 \mathbf{M} p a$ an

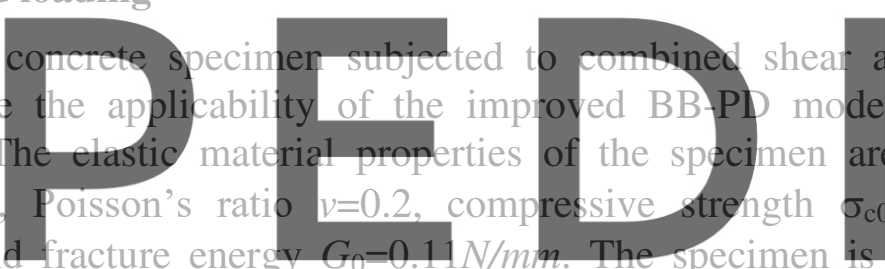
and tension
del for more
are gives as:
$\sigma_{c 0}=38.4 \mathrm{Mpa}$,
is discrized with a spacing of $\Delta=0.002 \mathrm{~m}$ and the material horizon is selected as $\delta=3 \Delta$.

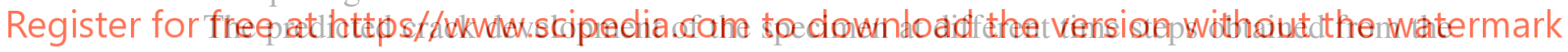
improved BB-PD model can be seen in Fig. 2. It indicates that the predicted cracks is initiated from the two notches and develop with an angle to the horizontal line at the early time steps. Then the crack trajectories deflect and extend along the horizontal line, which agrees well with the experimental observation [12] shown in the Fig. 3. In order to further verify the validation of the proposed model, the predicted force-displacement relations in vertical and horizontal directions of the specimen are presented in Fig. 4. It shows a good agreement between the results obtained from the proposed model and the experimental data from the literature [12]. Therefore, the validation of the improved BB-PD model as well as its applicability for complex combined loads is verified. 


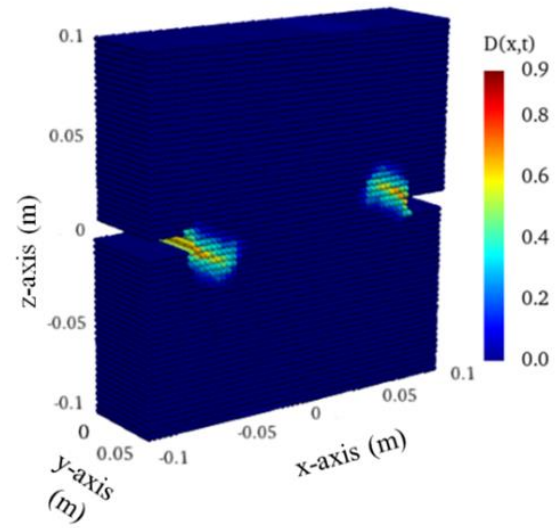

(a)

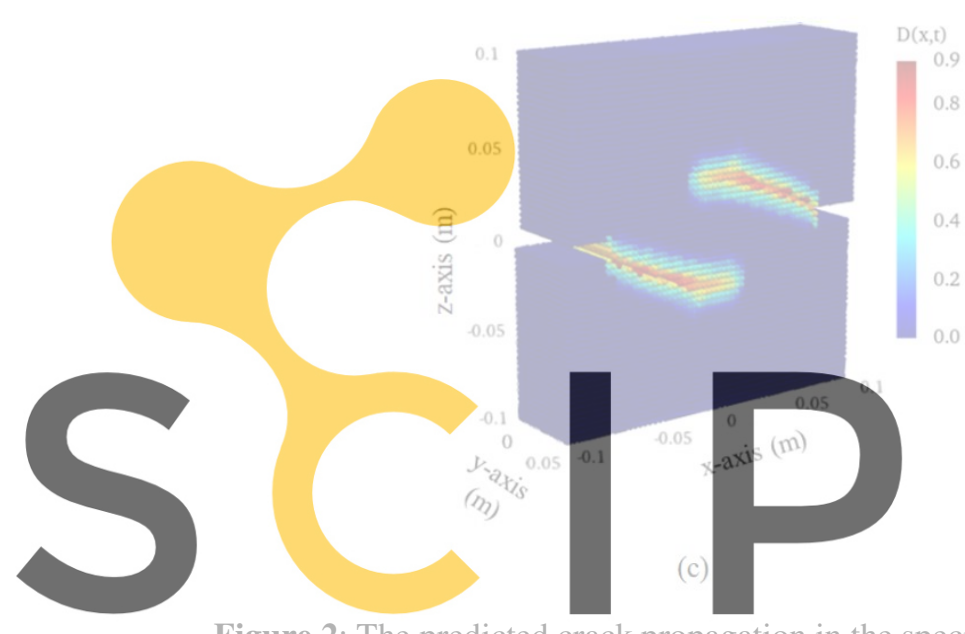

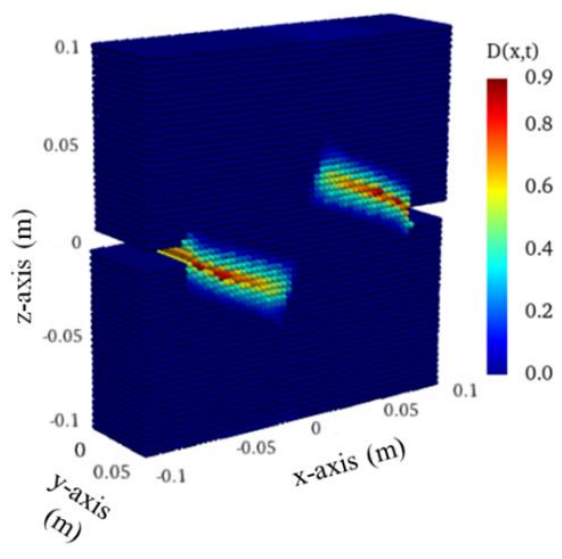

(b)

Figure 2: The predicted crack propagation in the specimen obtained from the proposed model at: (a) time step =

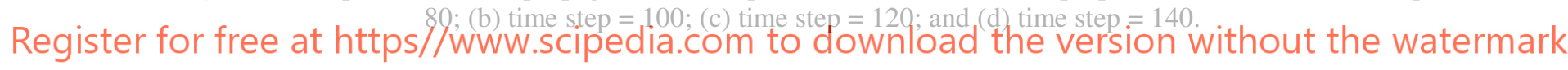
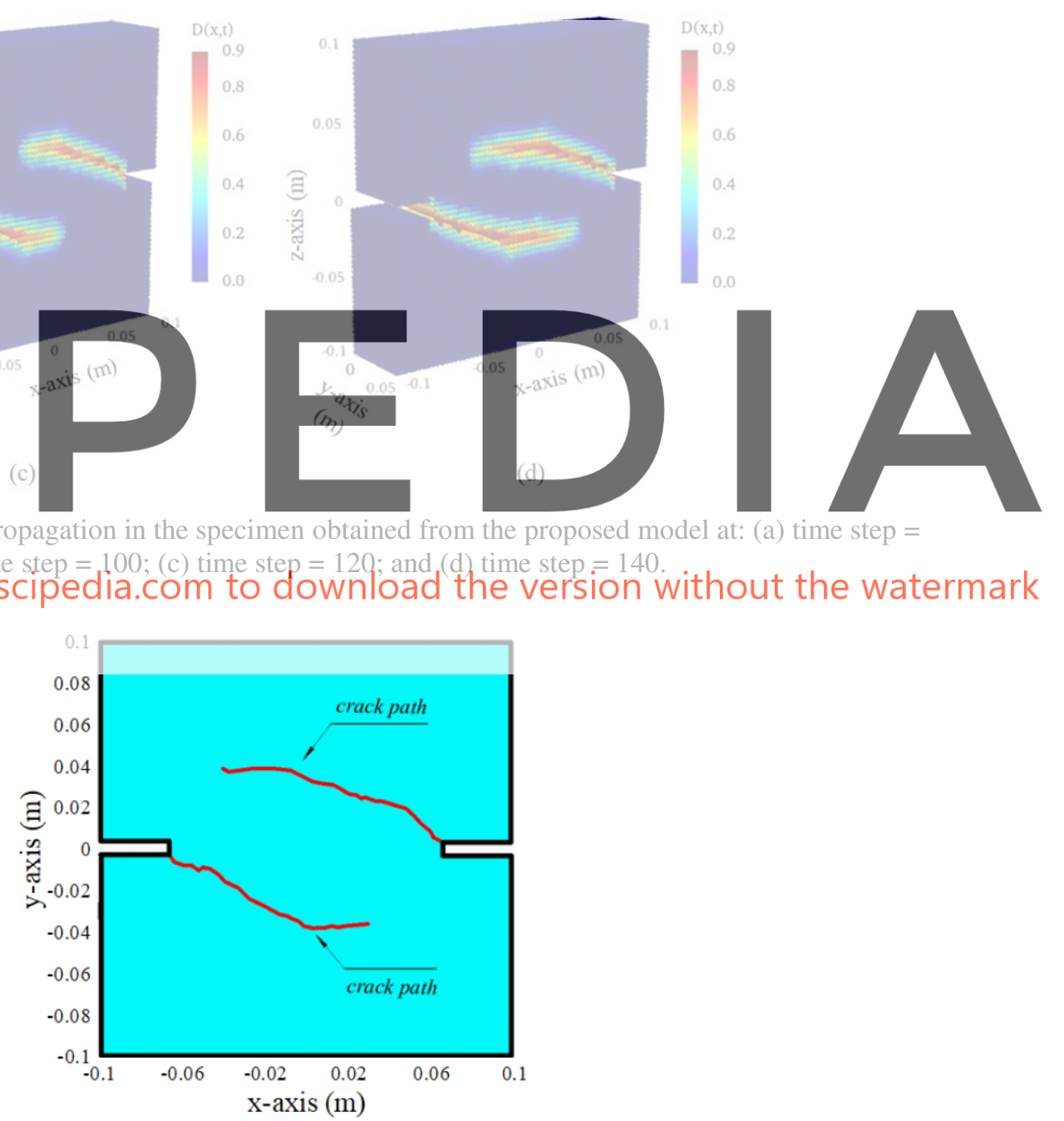

Figure 3: Experimental observation of the final crack growth paths in the double-edge-notched specimen [12]. 


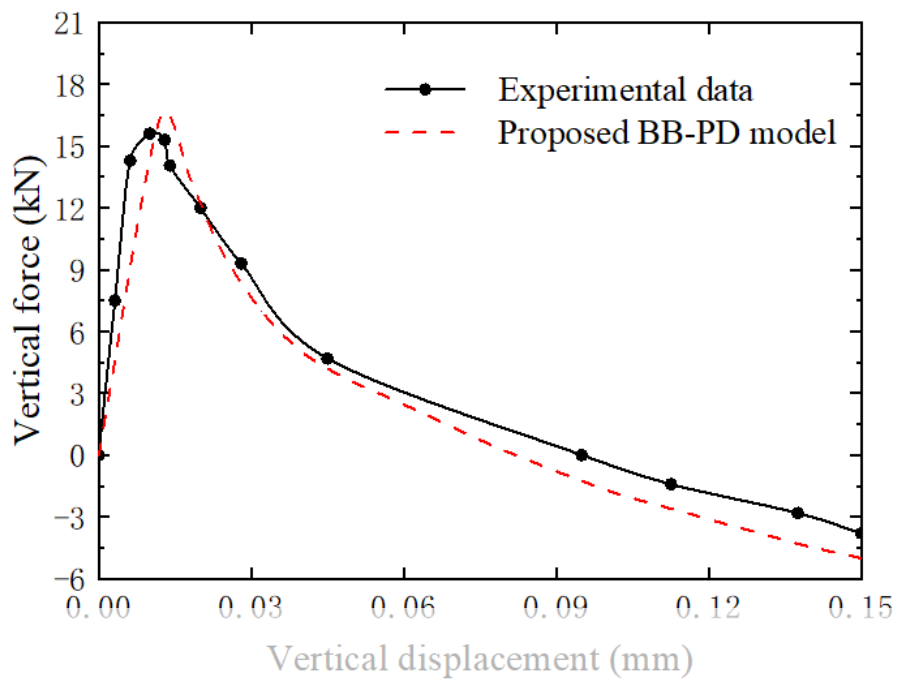

(a)
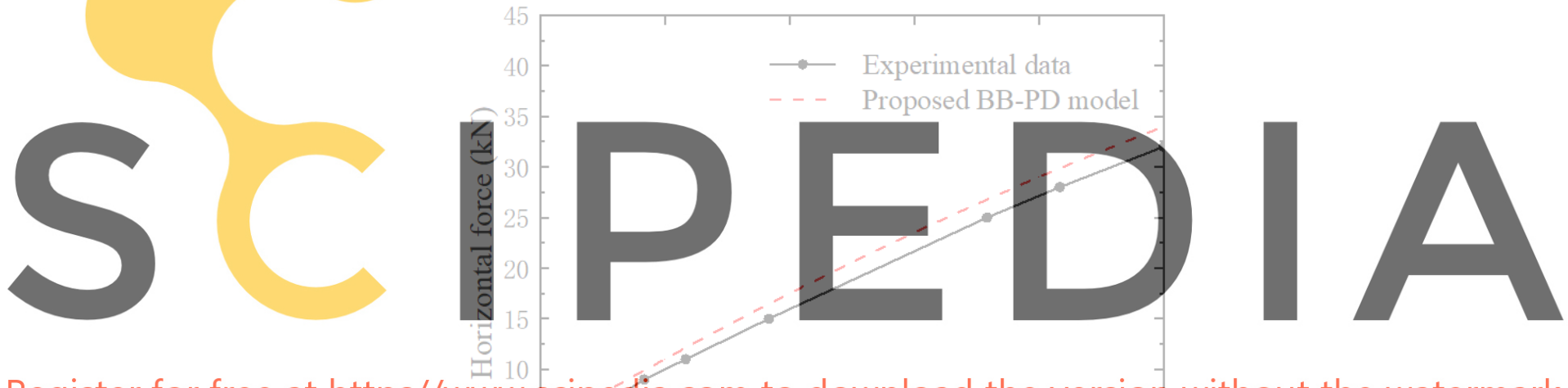

Register for free at https//www.scipédia.com to download the version without the watermark

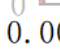

0.03

0.06

0.09

0.12

0.15

Horizontal displacement $(\mathrm{mm})$

(b)

Figure 4: Force-displacement relations of the double-edge-notched specimen under tension-shear combined loads in: (a) the vertical direction; and (b) the horizontal direction.

\section{CONCLUSIONS}

An improved micropolar bond-based peridynamic model based on Timoshenko beam theory is proposed in this paper. The mechanical behaviors of the bond between particles are reformulated with incorporation of the bond stretch, shear deformations and particle relative rotation angle, which eliminates the limitation of the Poisson's ratio and makes it suitable for complex discontinuous problems. Three peridynamic parameters related to the compressive, shear and bending stiffnesses of the beam, are introduced in the improved BB-PD model and 
keep the consistence between the strain energy of the peridynamic model and that of the continuum solid mechanics under arbitrary deformation fields. A new energy-based failure criterion is also proposed to simulate the progressive failure of quasi-brittle materials.

The validation of the improved BB-PD model is verified by comparing its predictions with available analytical solutions and experimental observations in known literatures. Some examples are conducted to demonstrate the applicability of the improved BB-PD model for tensile and tension-shear combined loading cases. Results show that the proposed model can solve the limitation for Poisson's ratio and capture the progressive failure of quasi-brittle materials under complex dynamic loads.

\section{ACKNOWLEDGMENTS}

The research has been supported by the National Natural Science Foundation of China (41922059 \& 51678438), the Shanghai Committee of Science and Technology (18DZ1205106), and the support from the Fundamental Research Funds for the Central Universities of China.

\section{REFERENCES}

[1] Silling, S. A. Reformulation of elasticity theory for discontinuities and long-range forces. Journal of the Mechanics and Physics of Solids (2000) 48(1):175-209.

[2] Silling, S. A., Epton, M., Weckner, O., Xu, J. and Askari, E. Peridynamic states and constitutive modeling. Jdurnal of Elasticty (2007) 88(2):151-184.
Warren, T. L., Silling, S. A., Askar, A., Weckner, O., Epton, M. A. and Xu, J. A non-
ordinary state-based peridynamic method to model solid material deformation and
fracture. International Journal of Solids and Structures (2009) 46(5):1186-1195.
Gerstle, W., Sau, N. and Silling, S. Peridynamic modeling of concrete structures. Nuclear engineering and design (2007) 237(12-13):1250-1258.

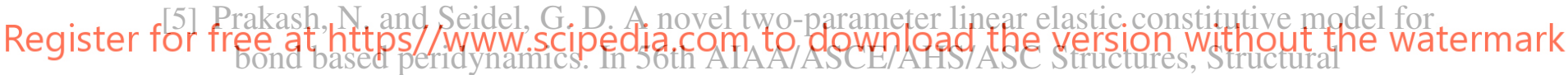
Dynamics, and Materials Conference (2015).

[6] Wang, Y., Zhou, X. and Shou, Y. The modeling of crack propagation and coalescence in rocks under uniaxial compression using the novel conjugated bond-based peridynamics. International Journal of Mechanical Sciences (2017) 128:614-643.

[7] Zhu, Q. Z. and Ni, T. Peridynamic formulations enriched with bond rotation effects. International Journal of Engineering Science (2017) 121:118-129.

[8] Diana, V. and Casolo, S. A bond-based micropolar peridynamic model with shear deformability: Elasticity, failure properties and initial yield domains. International Journal of Solids and Structures (2019) 160:201-231.

[9] Silling, S. A. and Askari, E. A meshfree method based on the peridynamic model of solid mechanics. Computers \& structures (2005) 83(17-18):1526-1535.

[10] Silling, S. A. and Bobaru, F. Peridynamic modeling of membranes and fibers. International Journal of Non-Linear Mechanics (2005) 40(2-3):395-409.

[11] Pavlovic, M. N. Fracture mechanics of concrete: applications of fracture mechanics to concrete, rock and other quasi-brittle materials. Engineering Structures (1996) 18(11): 887-888. 
[12] Nooru-Mohamed, M. B., Schlangen, E. and van Mier, J. G. Experimental and numerical study on the behavior of concrete subjected to biaxial tension and shear. Advanced Cement Based Materials (1993) 1(1): 22-37.
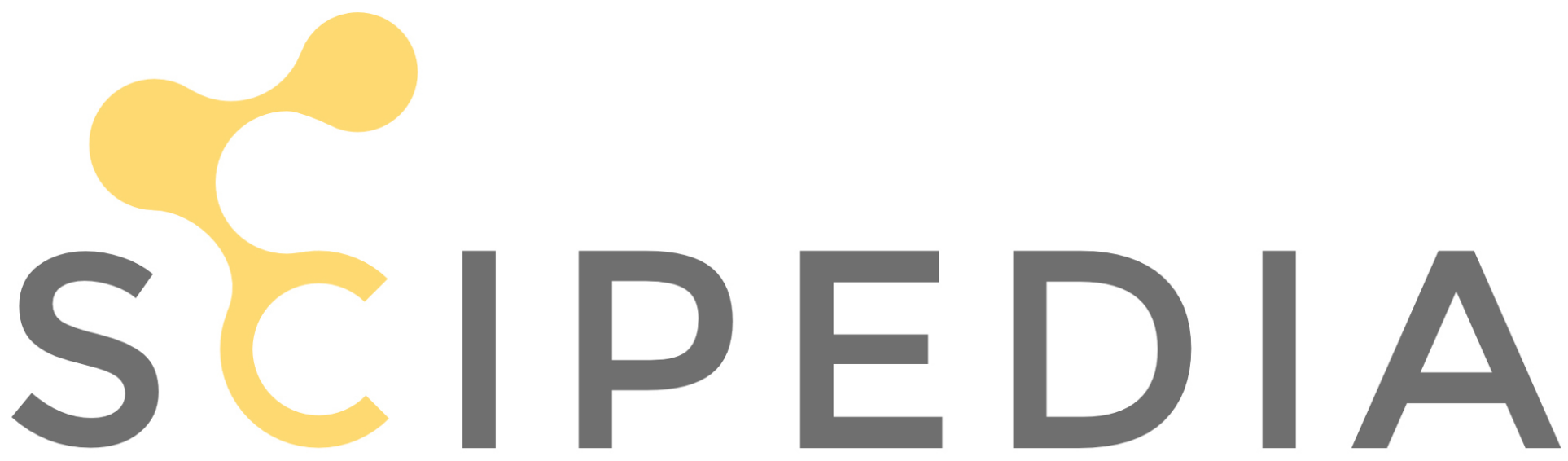

Register for free at https//www.scipedia.com to download the version without the watermark 